\title{
Prototype and Exemplar-Based Information in Natural Language Categories
}

\author{
Gert Storms, Paul De Boeck, and Wim Ruts \\ University of Leuven, Leuven, Belgium
}

\begin{abstract}
Two experiments are reported in which four dependent variables; typicality ratings, response times, category-naming frequencies, and exemplar-generation frequencies of natural language concepts, were predicted by two sorts of prototype predictors and by an exemplar predictor related to Heit and Barsalou's (1996) instantiation principle. In the first experiment, the exemplar predictor was compared to a prototype predictor calculated as in Hampton (1979). The four dependent variables were either predicted better by the exemplar measure than by the prototype predictor or the predictive value was about equal. In the second experiment, a new prototype predictor was calculated based on Rosch and Mervis' (1975) classic family resemblance measure. The results showed that the exemplar predictor accounted better for the dependent variables than Hampton's and Rosch and Mervis' prototype measures. The differences between the prototype measures were not significant. (๑) 2000 Academic Press
\end{abstract}

Key Words: natural language concepts; prototype theory; exemplar models; family resemblance.

The classical view of semantic concepts states that a concept can be described in terms of defining features that are singly necessary and jointly sufficient (e.g., Sutcliffe, 1993). Ample evidence has been provided against this view (see, e.g., Komatsu, 1992; Smith \& Medin, 1981, for overviews.) Two major alternative theories have been formulated: the prototype view and the exemplar view. In the prototype view (Rosch, 1975a, 1978, 1983; Rosch \& Mervis, 1975), it is assumed that categories are represented by a set of features which may carry more or less weight in the definition of the prototype, and categorization is

This research project was supported by Grant 2.0073.94 from the Belgian National Science Foundation (Fundamental Human Sciences) to P. De Boeck, I. Van Mechelen, and D. Geeraerts. Part of this research was conducted while the first author was visiting Doug Medin at Northwestern University. Their hospitality is gratefully acknowledged. We thank the following for discussion and advice in the course of the research and in the preparation of this manuscript: Lawrence Barsalou, Dedre Gentner, Lloyd Komatsu, Douglas Medin, Iven Van Mechelen, Ed Wisniewski, and one anonymous reviewer. All the data described in this manuscript can be obtained from the first author (in Excel files) upon simple request.

Address reprint requests to Psychology Department, University of Leuven, Tiensestraat 102, B-3000 Leuven, Belgium. E-mail address: Gert.Storms@psy.kuleuven.ac.be. based on whether an item possesses enough of these features. The exemplar view essentially states that a category is represented by particular instances that have previously been encountered. A new item is assumed to be judged an instance of a category to the extent that it is sufficiently similar to one or more of the instance representations stored in memory.

Different procedures have been proposed to derive prototype measures for natural language concepts (e.g., Hampton, 1979; Rosch \& Mervis, 1975; see also Barsalou, 1990). Also, different variants of the exemplar view have been presented in the literature, depending on the assumptions made about the number and nature of the instances stored, about the presence or absence of forgetting, and so on (Barsalou, 1990). In some theories it is assumed, for example, that every instance that is encountered is also stored (e.g., Reed, 1972), while in others it is assumed that only the most typical instances are stored (e.g., Rosch, 1975b) or that many instances are stored to varying degrees of completeness (see, Komatsu, 1992, for more details).

The evidence for exemplar models mostly consists of category learning data, obtained with tasks in which subjects learn new categories of 
artificial stimuli. In the context of natural language concepts, prototype and exemplar-based predictors have, to our best knowledge, not yet been compared.

In this paper, we want to focus on the extent to which category-based variables (typicality ratings, latency of category decision, exemplargeneration frequencies, and category-naming frequencies) derived from natural language concepts can be predicted from prototype measures and exemplar-based measures. Before describing the empirical work, we will elaborate on the differences between natural language concepts as they are used by adult language users on the one hand and concepts related to categories as they appear in laboratory studies in which subjects learn new categories of artificial stimuli on the other hand.

\section{EXEMPLAR AND PROTOTYPE MODELS FOR ARTIFICIAL CATEGORIES AND NATURAL LANGUAGE CONCEPTS}

In the past, many studies have compared prototype and exemplar-based predictions to explain categorization and category learning, mostly using perceptual stimuli (see Nosofsky, 1992). Only a few studies have been published in which the exemplars of the (new) categories to be learned were verbally described (e.g., a set of symptoms of fictitious patients in Medin, Altom, Edelson, \& Freko, 1982, or a set of features of fictitious persons in Hayes-Roth \& Hayes-Roth, 1977), and, although the stimuli were verbal, they are still somewhat artificial, as were the categories. As it cannot be taken for granted that the nature of categorization learning using artificial categories parallels the learning of natural language concepts, a generalization of the results to the mental representation of everyday natural language categories, such as trees, furniture, or games, in adult language users, seems problematic. Most people learn about birds and flowers erratically and from many different sources, contrary to the learning situation in a laboratory experiment where a category is learned in an explicit learning phase. Also, whereas subjects in an artificial category learning experiment are instructed to encode the exemplars in detail, there is no similar guarantee that people are equally careful in their ob- servation of real-world objects, and thus, that information is encoded in the same way (Malt \& Smith, 1984).

To our knowledge, the study of Heit and Barsalou (1996) is the only attempt to test a model compatible with most exemplar models in the context of natural language concepts. Heit and Barsalou proposed an instantiation model in which it is essentially assumed that people generate instantiations of a category to base category-related decisions on. More specifically, Heit and Barsalou predicted the typicality of lower level concepts (like, e.g., mammals) within a higher level concept animal from the typicality of instances of the lower level concept (like, e.g., dog, horse, cat) within the higher level concept animal.

In contrast with the exemplar view, much evidence in favor of the prototype view stems from natural language concepts. As Hampton (1979) and Rosch and Mervis (1975) demonstrated, prototype-based predictions succeed in explaining a considerable portion of the variance in typicality judgments for natural language concepts. Applying the exemplar models to natural language categories, however, is not straightforward. An important problem in order for the exemplar view to be tested for natural categories is that "it is not entirely clear what an exemplar representation is" (Komatsu, 1992, p. 507). At one extreme, an exemplar representation might be a family resemblance representation that abstracts across different specific instances. In this view, the concept fish may consist of the set of representations of trout, goldfish, shark, and so on, which are themselves abstractions. At the other extreme, exemplar representations may involve no abstraction at all, with representations consisting only of specific memory traces of particular previously encountered instances. (See, e.g., Medin, 1986, ${ }^{1}$

${ }^{1}$ Though the latter view may be more popular among researchers that have studied artificial category learning, one can doubt whether a theory which assumes no abstraction at all has ever been tested in any of the laboratory studies. In designing these experiments, researchers assume that the representation of the presented stimuli consists of the dimensions that they manipulate (e.g., color, form, size, and position of the stimulus), but if subjects do not abstract these dimensions from other information that may in prin- 
and for a strong critique of nonabstracting exemplar models, see Barsalou, Huttenlocher, \& Lamberts, 1998.)

Heit and Barsalou's (1996) instantiation principle described above is not explicit about exactly how exemplar information is stored in memory. The model assumes that categorybased decisions about concepts like birds, fish, mammals, etc. are based on information stored at the level of instantiations that are retrieved from memory (i.e., at the level of robin, trout, and horse). However, the proposed instantiation principle is equally compatible with a model that assumes no abstraction at all as with a model that assumes abstraction across specifically encountered examples at the level of the instantiated exemplars.

To summarize, in the context of natural language categories like fruits, vegetables, vehicles, etc., three different theoretical views may be distinguished depending on the levels at which abstraction does or does not take place. The first view assumes that no abstraction whatsoever takes place and that only memory traces of particular encountered instances are stored. Any category-related judgment is based on these memory traces, as no abstract information is stored with verbal concepts. The second view assumes that abstraction may take place, but only at a level lower than the concepts studied, that is, at the level of tomatoes in case vegetables are studied. The representation of the studied natural language concepts, like vegetables and vehicles, is comprised of lower level concepts like tomatoes and bikes, respectively. Finally, the third view states that abstraction (also) takes place at the level of the studied natural language concepts and that (characteristic) features of their exemplars are directly stored at this level. The latter view can be labeled the prototype view, and the first two

ciple be stored (like, e.g., the trial number and slight differences in illumination due to uncontrollable events), they might not be able to learn the categories. Thus, each training exemplar is, so to speak, a sort of a prototype consisting of a set of abstracted features that, in principle, can apply also to other exemplars that differ on some other, irrelevant, features (D. L. Medin, personal communication, May 19, 1997). views are exemplar views. Both of these exemplars views are compatible with Heit and Barsalou's (1996) instantiation principle.

The present paper describes two experiments in which two different prototype predictions and an exemplar prediction inspired by Heit and Barsalou's (1996) instantiation will be compared to predict category-based decisions for eight natural language concepts.

\section{EXPERIMENT 1}

In the first experiment, a first prototype predictor and the exemplar-based predictors will be compared for the four different category-related dependent variables. We will first motivate the choice of the four dependent variables and then elaborate on the prototype and exemplar-based measures, which will be used as predictors for the dependent variables.

\section{Typicality Ratings}

Typicality has been shown to be a very influential variable in a wide variety of cognitive tasks (Hampton, 1993; Malt \& Smith, 1984), such as speeded categorization (Hampton, 1979), inductive inference (Rips, 1975), productive tasks (Hampton \& Gardiner, 1983), priming effects (Rosch, 1975b), semantic substitutability (Rosch, 1977), and memory interference effects (Keller \& Kellas, 1978). According to the prototype view, variations in category typicality reflect differences in similarity to the prototype in terms of the features it shares with the prototype representation of the concept. The extent to which items are characterized by features that are important in deciding on category membership has been reported to be a good predictor by Hampton (1979), Malt and Smith (1984), and Rosch and Mervis (1975). According to the exemplar view, variations in category typicality reflect varying degrees of similarity to stored exemplars of the category. The instantiation principle, proposed by Heit and Barsalou (1996), can be adapted to account for typicality ratings within basic level categories like birds, fish, and mammals. In evaluating the typicality of a particular instance $X$ within a category $Y$, a subject might first generate one or more stored exemplars of the category $Y$. Next, the typicality of instance $X$ within $Y$ might be based on the 
similarity of instance $X$ toward the instantiated exemplars stored earlier under $Y$. In summary, both views explain typicality in terms of their basic assumptions about how categories are represented. Therefore, finding which of the two views best predicts typicality ratings may shed light on the representation of semantic categories.

\section{Response Times}

The second measure to be predicted by both models is response time in a speeded categorization task. A very robust finding in the literature on semantic verification tasks is that the time to verify category membership differs significantly among the members of the same category (Larochelle \& Pineau, 1994). This difference in response times can be explained both by the prototype model and by exemplar models. Following Hampton's (1979) assumptions about category prototypes, different characteristic features of the concept can be selected successively in time and a "Yes" response can be emitted as soon as the feature overlap between the stored features of the prototype and the features of a presented word reaches a certain threshold. Likewise, subjects can be assumed to give a "No" response as soon as the set of nonmatching features reaches a certain criterion. Hampton's results show that a considerable percentage of the variance in the response times can be accounted for by a prototype-based measure. (See also McCloskey \& Glucksberg, 1979.)

Exemplar-based predictions about response times in a speeded categorization follow a finding of Hines, Czerwinski, Sawyer, and Dwyer (1986), who found that good category members prime other category members (regardless of their association level), while medium category exemplars fail to produce semantic priming for within-category members. Based on these findings, it could be assumed in the exemplar model that a category member presented in a speeded categorization task might activate other exemplars based on a simple mechanism in which similar exemplars are retrieved first. If the number of retrieved items that are directly stored exemplars of the category (i.e., good members of the category) reaches a threshold value, then the subjects can give a "Yes" response. If, however, the number of retrieved items that are not stored exemplars reaches a critical value first, then a "No" response is assumed to be emitted. It follows from this hypothesized process that, within a category, response times should decrease with increasing similarity toward stored good exemplars of the category. (See Nosofsky \& Palmeri, 1997, for another exemplar-based model of response times.)

\section{Category-Naming and Exemplar-Generation Frequency}

Category-naming and exemplar-generation frequency are, in a way, each other's opposite: In an exemplar-generation task, participants are given the category label and are asked to name exemplars, while in a category-naming task, participants are given exemplars and they are asked to name the category.

As for category naming as a dependent variable, the fact that some exemplars of a category are labeled with the category name more frequently than other exemplars can be explained by the exemplar-based models when assuming that the link between a category and an exemplar is of a probabilistic nature. The prototype model can account for this finding in assuming that the feature pattern associated with the given exemplar somehow activates the feature pattern of the category (which then allows the subject to retrieve the category label) and that this is more likely the more the feature patterns of the exemplar and of the category resemble each other. The difference in both views is whether or not the activation goes through a feature pattern and from there to the category label or goes directly to the category label. However, exactly how this activation would work is not clear. Besides, there is another complicating factor in that exemplars can be linked to more than one category label. Neither the prototype nor the exemplarbased models have dealt in an explicit way with the problem of overlapping categories.

The situation is even less clear in the exemplar-generation task. Exemplar-based models assume that categories are learned by associating the category label to given exemplars and by evaluating the similarity of new stimuli to stored exemplars. Thus, these models have to be 
extended to account for the fact that subjects can easily do the reverse, that is, generate exemplars when given a category label. The prototype view again has to rely on a feature-based activation process in which the feature pattern associated with the category somehow activates the feature pattern of category exemplars.

In sum, neither the prototype nor the exemplar-based model is very clear on the process underlying the responses in a category-naming task and in an exemplar-generation task. Given that both types of models are not really elaborated with the types of tasks under consideration, it is useful to collect data of this kind to help to develop the models further.

\section{Prototype and Exemplar Based Predictors}

In this first experiment, we compared an exemplar-based predictor with a prototype predictor. We considered a version of the exemplar view in which subjects use the stored information about the "best exemplars." Note that our conception differs somewhat from the instantiation model proposed by Heit and Barsalou (1996). Heit and Barsalou assume that subjects instantiate a concept with only one single exemplar, possibly a different one depending on the subject, that is, the instantiation that first comes to their mind, while we assume that subjects can instantiate a concept using more than one exemplar. Though the exemplar activation process is not observable, it can be assumed that it is possible to derive an approximation of the sampling distribution of the concept instantiations over subjects from the results of an exemplar-generation task. Since we had a list of concept instantiations from an earlier exemplar-generation task (Storms et al., 1996), we could evaluate the impact of taking into account different numbers of exemplars in deriving the exemplar-based predictor. Thus, different predictors could be constructed by summing the similarity toward an increasing number of "best" exemplars of the category, "best" meaning the most frequently generated exemplars, weighted for their production frequency.

The prototype prediction used in Experiment 1 is derived using Hampton's (1979) procedure. More specifically, characteristic features of the concepts were obtained from Hampton's study. Next, the applicability of each characteristic feature of the category is evaluated for different items and a sum of these feature applicabilities is used to predict category-related decisions for the corresponding items.

\section{Method}

We will first give an overview of the material used in this experiment. Next, we will describe the different tasks used to gather data on which the dependent and the independent variables were based.

\section{Material}

All concepts and items used were in Dutch and all subjects were native Dutch speakers. Eight common categories, previously studied by Hampton (1979), were used in this experiment: kitchen utensils, furniture, vehicle, sport, fruit, vegetable, fish, and bird. The set of categories contained natural kinds, artifacts and activities, with natural kinds and nonnatural kinds equally balanced (four of each), which was desirable given the potential effects of this nature of the concepts studied (e.g., Malt \& Johnson, 1992). For each of these categories, a list of 36 items was selected from an exemplar generation study (Storms, De Boeck, Van Mechelen, \& Ruts, 1996): 24 presumed exemplars and 12 nonexemplars that were related to the category. The exemplar set always included the most frequently generated exemplar, but also items with presumably varying degrees of typicality within the categories. The 12 related nonexemplars of each of the eight categories were selected from the results of an exemplar-generation task, where subjects were asked to write down exemplars of superordinates of the eight categories, excluding the category itself (e.g., of food that is not fruit, of animals that are not fish, etc.).

Features of the eight categories were taken from Hampton's study. Hampton gathered these features by interviewing 32 undergraduate studies extensively. The interview consisted of a first part, in which the participants gave free descriptions of the eight concepts, and a second part, in which they were given seven questions to further encourage them to generate as many features as possible. Features generated by less 
than $25 \%$ of the participants were excluded. For the eight categories, respectively 13, 11, 12, 14, $13,9,16$, and 12 features were selected to derive the prototype prediction. (Note that the participants in Hampton's study had English as their mother tongue, while our participants were Dutch speaking. However, data gathered recently in the context of another study, where the same eight categories were used, indicated that the feature set for the Dutch translation of the eight concept labels yielded virtually identical feature sets.)

\section{Procedure}

To derive the prototype and exemplar-based predictor variables, two different tasks were given to different groups of subjects: a similarity rating task and a feature applicability task.

\section{Similarity Rating Task}

Participants. Two hundred and fifty students from the University of Leuven participated for course credit.

Procedure. Participants indicated the similarity of 36 listed items (24 presumed exemplars and 12 related nonexemplars) toward a keyword. The similarity judgments were given on a 10-point rating scale (ranging from 1 "no similarity at all," to 10 "highly similar"). No indication was given as to the point of view from which similarity had to be considered. As key words, the 25 most frequently generated exemplars of the category were used. (The 10 most frequently generated exemplars for every category are given in the Appendix). The participants each received eight lists of 36 items, one per concept, in order to judge the similarity of the items of each list to one of the 25 key words for that list, that is, one of the 25 most frequently generated exemplars. Key words were distributed randomly over participants, with the restriction that all participants rated all item lists for their similarity to only one key word. Note that the 25 most frequently generated exemplars of the category that were used as target stimuli in the similarity rating task usually contained the majority of, but not all of, the 24 category exemplars of the item list. (The 24 items contained the 10 most frequently generated exemplars, but the remaining 14 items were selected so as to cover the whole range of varying typicalities in the concept.) The task took approximately $30 \mathrm{~min}$.

\section{Item by Feature Applicability (Matrix Filling) Task}

Participants. Eighty students from the University of Leuven participated for course credit.

Procedure. Participants were given a matrix where the rows were labeled with the 36 items and where the columns were labeled with the category features taken from Hampton (1979). They were asked to fill out all entries in the matrix with a 1 or a 0 to indicate whether or not a feature was considered present in the item corresponding to the row of the entry. Completion of the applicability matrix took about 50 $\min$.

Four other tasks were administered in the first experiment to obtain the four dependent variables.

\section{Typicality-Rating Task}

Participants. Ten students from the University of Leuven participated for course credit.

Procedure. The participants received standard instructions for typicality ratings. A 7 -point rating scale was used (ranging from -3 for very atypical or unrelated items to +3 for very typical items). All participants rated typicality for the item sets of all eight categories. The task took approximately $30 \mathrm{~min}$.

\section{Speeded Categorization Task}

Participants. Eighteen last-year psychology students from the University of Leuven participated voluntarily.

Procedure. The participants were seated in front of a computer screen and read the instructions from the screen. They were asked to perform a speeded categorization task for nine categories (the eight concepts studied plus one concept used to acquaint the participants with the procedure). They saw the name of a category printed in bold in the middle of the screen and were instructed that words would appear right under the category name. They were asked to decide as quickly as possible whether or not the word shown under the category name belonged to the category. In order to avoid ex- 
treme response bias, four additional nonmembers of the categories were included. As a consequence, the item set of every concept comprised 24 members and $16(12+4)$ nonmembers. Assignment of the two response keys, labeled "Yes" and "No," was counterbalanced over participants. The participants kept the index fingers of both hands on the response keys at all times, except during the pauses in between two categories. The experiment started with a practice category (flowers). Participants were asked to write down the words they had responded to incorrectly, right after the completion of every category. They were told that doing the task as fast and as accurate as possible was much more important, however, than trying to remember incorrect responses. As in Hampton (1979), all response times for trials marked as mistakes by the participants and all response times of "No" answers for members were discarded. Participants were informed that they could pause in between two categories. The order of the eight categories was determined following a Latin square design. The experiment typically took 35 to $40 \mathrm{~min}$ to be completed.

\section{Category Naming Task}

Participants. Three hundred and sixty students from the University of Leuven participated voluntarily.

Procedure. The participants were given a list of eight items, each being one of the 36 items associated with a different category (as there were eight categories studied), either as a member or as a nonmember. The participants were asked to write down, for each item, the first category they thought the item belonged to. The task was administered collectively at the beginning of a class and took only a couple of minutes. There were 36 different lists of eight items and ten different participants completed every list.

\section{Exemplar Generation Task}

Participants. Fifteen graduate students of the Psychology Department participated voluntarily.

Procedure. The participants were given a booklet in which they had to write down ten different exemplars of each of the eight concepts studied. They were asked to write the exemplars in the order that they thought of them.

\section{Results}

\section{Reliability}

The reliability of all the data that we used in this paper was evaluated by the split-half correlations corrected with the Spearman-Brown formula, with the halves referring to halves of the subjects that completed the task in question. All reliability coefficients are derived over the 36 items associated with a category. Similarity ratings with the 25 best exemplars of each category had a mean reliability estimate of .94 (with only 15 out of the 200 ratings having an estimated reliability below .90). Reliabilities were estimated for all features (i.e., all columns) in the applicability matrices of the eight concepts. Mean estimates of $.96, .97, .98, .96, .88$, $.93, .91$, and .95 were found for fruit, birds, vehicles, sports, furniture, fish, vegetables, and kitchen utensils, respectively. Estimated reliabilities of the typicality ratings of all categories were above .985 . For the response times, estimates of the reliability of the response times were $.49, .89, .81, .64, .87, .89, .41$, and .84 , respectively. For category naming, reliabilities were all well over .93, except for kitchen utensils (.85). Finally, the estimates for the data of the exemplar-generation task were $.92, .85, .95$, $.92, .97, .95, .83$, and .89 , respectively.

Prototype predictions. The prototype predictions were calculated as in Hampton (1979). The procedure described below is also illustrated in Fig. 1. Recall that the applicability matrix has frequencies as its entries, corresponding to the number of participants (out of 10) that judged the corresponding feature applicable to the corresponding item. Different prototype predictions were calculated based on the item by feature applicability matrix. A first measure used no weighting of the features. Thus, the prediction here consisted simply of summing over the different features, the number of subjects that credited the item with the corresponding feature. Three other measures were derived by first weighting the features before 


\section{Step1: Filling up the Item by Feature Matrix}

Features Generated for Concept
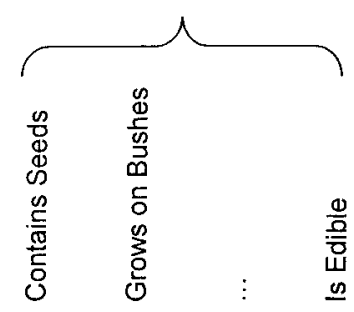

$\begin{array}{cccccc}\begin{array}{c}\text { Strawberry } \\ \text { Pineapple }\end{array} & 3 & 10 & \ldots & 10 & 10 \\ \vdots & 1 & 1 & \ldots & 10 & \text { Number of Subjects out of } 10 \text { that } \\ \text { Patato } & 1 & 3 & \ldots & 10 & \begin{array}{c}\text { Credited "Pineapple" with the Feature } \\ \text { "is Edible" }\end{array}\end{array}$

Step2: Calculating the Prototype Predictions

$\begin{array}{ccc}\text { Strawberry } & 3+10+\ldots+10= & 86 \\ \text { Pineapple } & 1+1+\ldots+10= & \mathbf{8 8} \\ \vdots & & \\ \text { Patato } & 1+3+\ldots+10= & 39\end{array}$

FIG. 1. Calculating Hampton's (1979) prototype prediction.

summing the applicability frequencies. The weights were based (1) on the rated feature importance for defining the concept, (2) on ratings of how characteristic the features are for the concept, and (3) on the production frequency of the features. All three weights were taken from Hampton (1979). Given the a priori nature of the weights, no (estimated) free parameters were used.

Exemplar prediction. To our best knowledge, there are no guidelines to be found in the literature concerning the number of exemplars that are activated by a concept label for natural language categories. Therefore, several exemplar-based predictors were tried, which differed from each other in that an increasing number of exemplars were assumed to be activated. A comparison of these predictors enabled us to evaluate the effect of enlarging the set of exemplars.

Correlations were calculated between 25 different exemplar predictors and the four dependent variables. The 25 exemplar predictors were constructed based on an ordering of exemplars derived from generation frequency. Note that these frequencies were determined based on the results of an earlier study described in Storms et al. (1996). The first predictor simply consists of the rated similarity toward the first ordered exemplar of the category. For the second predictor, similarity ratings toward the two best-ordered exemplars were summed. The two terms of the sum were weighted using the production frequency of the corresponding items. The remaining predictors were constructed by adding each time the similarity ratings toward the next most frequently generated exemplar of the category, weighted by the production frequency. Note that no (estimated) free parameters were used in the exemplar-based predictors. Furthermore, we remind the reader that, in the similarity-rating task on which the exemplar prediction is based, no indication was given as to the point of view from which similarity had to be considered. However, due to the composition of the item list (which contained 24 members of a particular category and 12 related nonmembers), it is plausible that participants have rated similarity based at least partially on the features related to the category.

Prediction of the four dependent variables. For each of the different prototype and exem- 
TABLE 1

Correlations of the Four Dependent Variables with the Hampton Prototype Predictors for the Category Members

Correlation of Hampton prototype predictors versus

\begin{tabular}{|c|c|c|c|c|}
\hline Concept & Typicality & $\begin{array}{c}\text { Mean response } \\
\text { times }\end{array}$ & $\begin{array}{c}\text { Category-naming } \\
\text { frequencies }\end{array}$ & $\begin{array}{c}\text { Exemplar-generation } \\
\text { frequencies }\end{array}$ \\
\hline Fruits & $.55^{* *}$ & -.29 & .20 & .10 \\
\hline Birds & $.94 * *$ & $-.66^{* *}$ & $.74 * *$ & $.55^{* *}$ \\
\hline Vehicles & $.78^{* *}$ & $-.57 * *$ & $.71 * *$ & $.47 *$ \\
\hline Sports & $.92 * *$ & $-.66^{* *}$ & $.72 * *$ & $.40 *$ \\
\hline Furniture & $.79 * *$ & $-.76^{* *}$ & $.64 * *$ & $.51^{* *}$ \\
\hline Fish & $.68 * *$ & $-.77 * *$ & $.44^{*}$ & .25 \\
\hline Vegetables & $.66^{* *}$ & $-.53 * *$ & .32 & .03 \\
\hline Kitchen utensils & $.62 * *$ & $-.72 * *$ & .23 & $.35^{*}$ \\
\hline Mean & $.79 * *$ & $-.64 * *$ & $.54 * *$ & .34 \\
\hline
\end{tabular}

Note. ${ }^{*} p<.05, * * p<.01$. All others not significant.

plar-based measures, all analyses on the data were done with and without weights to determine predictor variables. However, concerning the prototype predictor, only the results for the unweighted prototype predictor will be given, since none of the three different feature weightings improved the prediction of the unweighted prototype measure. (Averaged over the four dependent variables and over the eight concepts, the unweighted prototype did explain a larger proportion of the variance than the weighted prototypes, but the difference was small and not significant.) In the remainder of the article, we will refer to the unweighted sum of feature applicability frequencies with the term "prototype prediction." For the exemplar-based predictors, only results for the weighted versions will be reported, since here the weighting did improve the prediction somewhat.

The prototype and the exemplar-based measures were correlated with all four dependent measures. All correlations were based on the 24 members of the categories only, excluding the related nonmembers of the categories from the analyses. Only for typicality ratings the analyses were also done for the complete list of 36 items. It would be problematic for response times to include nonmembers, as fast "Yes" responses are observed for typical members and slow "Yes" responses are emitted for atypical members; while fast "No" responses are ob- served for totally unrelated nonmembers and slow "No" responses are emitted for related nonmembers (Hampton, 1979). For the exemplar-generation task, evidently, almost all of the nonmembers were never generated as exemplars of the categories, and in the categorynaming task, the concept label was almost never given in response to nonmembers of the categories. Therefore, there was no variation in 12 of the 36 items, yielding an odd kind of distribution. Furthermore, by excluding the nonmembers, it is even more difficult to obtain high predictive levels. Thus, if the predictive level is high all the same, the evidence in favor of the model is considerably stronger.

Prototype predictions. Table 1 shows the correlations of the prototype predictor with the four dependent variables for the eight concepts studied. The prototype predictor is better in explaining the typicality ratings than the other dependent variables. All values are significant at the $\alpha=.01$ level. Also, the correlations for the response times are quite high and significant at the $\alpha=.01$ level for all concepts except fruits. Finally, the correlations for the generation frequencies and for the category-naming frequencies are somewhat lower and significant for only five concepts.

The exemplar-based predictors. In Fig. 2, the correlations for the 25 exemplar predictors are displayed graphically. Thirty-two diagrams 
Typicality
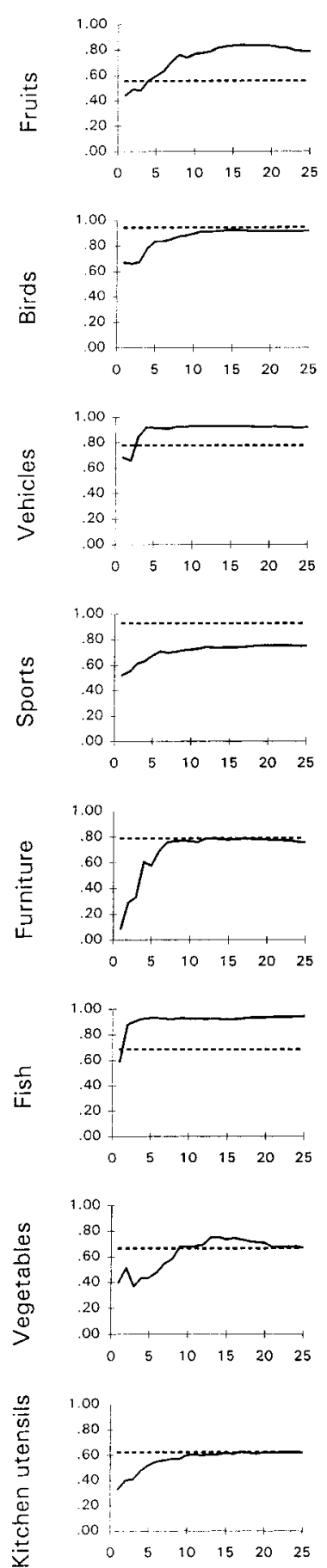

Speeded categorisation frequencies
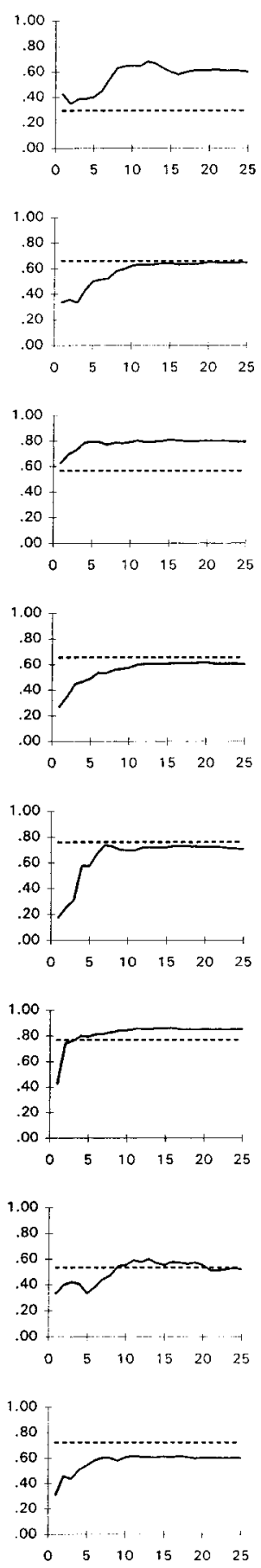

\section{Category naming frequencies}
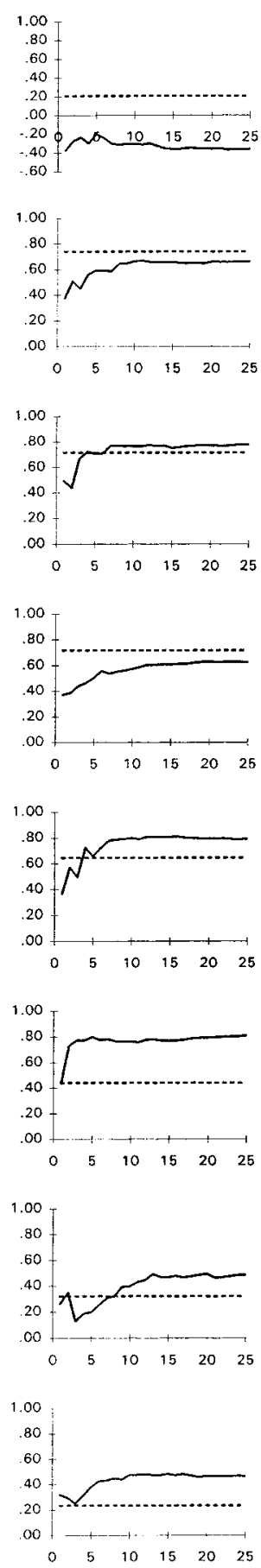

Exemplar Generation Frequencies
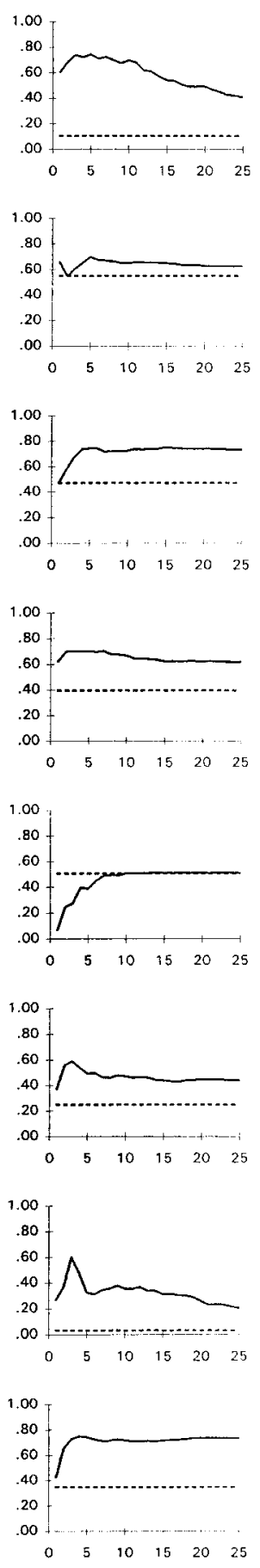

FIG. 2. Correlations of typicality, mean response times, category-naming frequencies, and exemplargeneration frequencies with the sum of 1 to 25 exemplar-based predictions for the category members only. (The dotted line gives the correlation with the prototype predictions.) 
TABLE 2

Correlations of the Four Dependent Variables and Prototype Predictors with the Exemplar Predictors Based on

Weighted Sum of Similarities Toward the Ten Most Frequently Generated Exemplars for the Category Members

Correlation weighted sum of similarities toward the ten most frequently generated exemplars versus

\begin{tabular}{lccccc}
\cline { 2 - 5 } \multicolumn{1}{c}{ Concept } & Typicality & $\begin{array}{c}\text { Mean response } \\
\text { times }\end{array}$ & $\begin{array}{c}\text { Category-naming } \\
\text { frequencies }\end{array}$ & $\begin{array}{c}\text { Exemplar-generation } \\
\text { frequencies }\end{array}$ & $\begin{array}{c}\text { Hampton prototype } \\
\text { predictors }\end{array}$ \\
\hline Fruits & $.77^{* *}$ & $-.65^{* *}$ & $.59^{* *}$ & $.70^{* *}$ & $.59^{* *}$ \\
Birds & $.89^{* *}$ & $-.62^{* *}$ & $.77^{* *}$ & $.65^{* *}$ & $.91^{* *}$ \\
Vehicles & $.93^{* *}$ & $-.79^{* *}$ & $.80^{* *}$ & $.72^{* *}$ & $.88^{* *}$ \\
Sports & $.72^{* *}$ & $-.58^{* *}$ & $.53^{* *}$ & $.67^{* *}$ & $.70^{* *}$ \\
Furniture & $.76^{* *}$ & $-.70^{* *}$ & $.43^{*}$ & $.51^{* *}$ & $.79^{* *}$ \\
Fish & $.93^{* *}$ & $-.84^{* *}$ & $.63^{* *}$ & $.47^{* *}$ & $.69^{* *}$ \\
Vegetables & $.68^{* *}$ & $-.55^{* *}$ & $.47^{*}$ & $.36^{*}$ & $.49^{* *}$ \\
Kitchen utensils & $.60^{* *}$ & $-.60^{* *}$ & .24 & $.72^{* *}$ & $.56^{* *}$ \\
Mean & $.82^{* *}$ & $-.68^{* *}$ & $.59^{* *}$ & $.61^{* *}$ & $.74 * *$ \\
\hline
\end{tabular}

Note. ${ }^{*} p<.05 .{ }^{* *} p<.01$. All others not significant.

show the correlations for the exemplar-based predictors of the eight concepts (corresponding to the eight rows) and the four dependent variables (corresponding to the four columns). The abscise of each of the diagrams corresponds to the increasing number of best exemplars taken into account in the predictor. The ordinate corresponds to the value of the correlation found between the dependent variable and the predictor. (Note that the correlation values in the second column, corresponding to the response times, are negative.) To ease the comparison of the exemplar-based predictors with the prototype predictor, a horizontal line is drawn in each graph, indicating the level that corresponds to the correlation of the prototype predictor.

It can be seen clearly that the different diagrams are similar in showing that the exemplar predictors improve when taking into account more exemplars. The improvement is strong when comparing the predictors based on up to seven exemplars. However, the improvement decreases as the number of exemplars taken into account increases. There is almost no improvement in the predictive power after adding more than ten exemplars. The first five columns of Table 2 show the correlations of the exemplar predictor based on the weighted sum of the 10 most frequently generated exemplars with the four dependent variables for the eight concepts studied.
It is remarkable how similar the diagrams in the first two columns of Fig. 2 are, meaning that the predictions of the typicality ratings and of the speeded categorization task are very similar. As can be seen in Table 2, the correlations of the exemplar-based measure for the 10 most frequently generated exemplars correlated significantly with typicality and with response times for all eight concepts $(p<.01)$. In general, the predictions for the typicality-rating task are a little better, partly because the response times are less reliable than the typicality ratings. The resemblance of the result patterns of the response times and of the rated typicalities is higher for a concept the more reliable the response times of the concept are. In most of the diagrams, the exemplar-based measure predicts the dependent variable better or equally good as the prototype predictor, with the exception of the typicality ratings for sports and the response times for kitchen utensils, which were somewhat better predicted by the prototype measure.

A similar pattern of results showed up for the prediction of the category-naming frequencies. Again the exemplar prediction was better than the prototype predictor in the majority of the concepts studied (with the exception of sports and of furniture). The correlations shown in Table 2 (based on the weighted sum of the 10 "best" exemplars) was significant $(p<.05)$ for all concepts except kitchen utensils. Finally, for 
the exemplar-generation frequencies, the exemplar-based measure is clearly superior to the prototype predictor. The latter reaches the level of the exemplar predictor only for furniture. The values in Table 2 are significant for all eight concepts $(p<.05)$.

An important consideration for interpreting the correlations of the two predictors with the dependent variables is whether the prototype predictor and the exemplar-based predictor can be differentiated. The last column of Table 2 shows the correlations between the prototype predictors and the exemplar predictors based on weighted sum of similarities toward the ten most frequently generated exemplars. The values show that there is some overlap, but the two predictors are not at all indistinguishable. Only for the concepts birds and vehicles, both predictors are very much the same. Averaged over the eight concepts, the exemplar-based predictor and the prototype predictor have $51 \%$ of their variance in common. The percentage of common variance does not increase when taking similarities toward all 25 exemplars into account.

To obtain a more detailed view of the impact of the predictors in explaining the dependent variables for the different concepts, an analysis of variance (ANOVA) with a split-plot factorial design (Kirk, 1982) was conducted, where the eight concepts function as blocks, where the predictor variables (prototype and exemplarbased predictors) and the four dependent variables (typicality ratings, response times, category naming, and exemplar generation frequencies) are within-block factors, and where the sort of concepts (natural and nonnatural kinds) is a between-block factor. The dependent variable in the ANOVA was the percentage of explained variance (the square of the correlations shown in Tables 1 and in the second through the fifth column of Table 2).

The analysis yielded a significant effect of the dependent variable factor $(F(3,18)=12.42$, $p<.01)$. A posteriori contrasts revealed that the typicality ratings could be predicted better than the other three dependent variables $(t(18)=$ $5.43, p<.01)$ and that the response times from the speeded categorization task could be predicted better than the exemplar-generation fre-
TABLE 3

Correlations of Typicality with the Hampton Prototype Predictors and the Exemplar Predictors Based on Weighted Sum of Similarities for the Complete Set of 36 Items: Category Members and Nonmembers

\begin{tabular}{lcc}
\hline & \multicolumn{2}{c}{ Correlation of typicality versus } \\
\cline { 2 - 3 } \multicolumn{1}{c}{ Concept } & $\begin{array}{c}\text { Hampton prototype } \\
\text { predictors }\end{array}$ & $\begin{array}{c}\text { Exemplar } \\
\text { predictors }\end{array}$ \\
\hline Fruits & .97 & .97 \\
Birds & .98 & .98 \\
Vehicles & .97 & .96 \\
Sports & .98 & .85 \\
Furniture & .86 & .93 \\
Fish & .98 & .99 \\
Vegetables & .90 & .96 \\
Kitchen utensils & .85 & .77 \\
Mean & .96 & .95 \\
\hline
\end{tabular}

Note. All correlations are significant at the $p<.001$ level.

quencies and the category-naming frequencies $(t(18)=2.60, p<.01)$. Though the categorynaming frequencies showed a larger percentage of explained variance than the exemplar-generation frequencies, the difference was not significant. Furthermore, the interaction between the predictor factor and the dependent variable factor was also significant. Contrasts for interactions showed that the exemplar-based measure differed only significantly from the prototype measure in predicting the exemplar-generation frequencies $(t(18)=6.68, p<.01)$, with the exemplar-based measure predicting the frequencies better. All other effects and interaction effects were not significant, though the difference between the exemplar-based measure and the prototype measure approached significance for the typicality ratings $(t(18)=1.42, p<.10)$, with the exemplar-based measure yielding better predictions.

As mentioned previously, the results of the typicality ratings can also be analyzed when including the related nonmembers of the categories. Table 3 shows the correlations between the two predictor variables and the typicality ratings based on the complete set of 36 items for the eight concepts studied. These correlations are a lot higher than the correlations based on the members only (see Tables 1 and 2). These 
differences can easily be explained given a restriction of range in the analyses excluding nonmembers. An analysis of variance with a splitplot factorial design was conducted, with the eight concepts as blocks, the sorts of concepts (artifacts versus natural kinds) as a betweenblocks factor, and the two predictor variables (prototype measure and exemplar-based measure with 10 exemplars) as a within-blocks factor. The percentage of variance accounted for was again used as the dependent variable in the analysis. On the average, there was almost no difference in explained variance between the two predictor variables ( 87 and $86 \%$ for the prototype and the exemplar-based predictor, respectively). Though the mean percentage of explained variance was 93 and $81 \%$ for the natural kind concepts and for the artifact concepts, respectively, the difference was not significant.

\section{Discussion}

First of all, the main idea of an instantiation principle, proposed by Heit and Barsalou (1996) to account for typicalities of superordinate natural language categories (like birds, fish, mammals, etc.) within higher level concepts (like animals) was shown to work well in predicting typicalities of exemplars within these superordinate categories as well. The correlations of the dependent variables with the predictions based on the exemplar model were rather high. All but one of the 32 correlations ( 8 concepts by 4 dependent variables) were significant. Note, however, that the exemplar model proposed here allowed that a concept can be instantiated with more than one exemplar, whereas Heit and Barsalou's (1996) model assumes only one instantiation. For a detailed comparison of Heit and Barsalou's model and the model proposed here, see Storms, De Wilde, De Boeck, and Ruts (1999).

The results showed also very clearly that the predictive power of the exemplar-based measure increases when more exemplars are taken into account. However, this increase was not linear. The gain in predictive power slows down from seven exemplars on and on the average no improvement is found beyond ten exemplars.

Heit and Barsalou (1996) have not compared the predictions of the instantiation principle with predictions derived from prototype theory. The results obtained in our experiment suggest that, although for all four dependent variables the exemplar-based predictions were on the average better than the prototype predictions, the difference was not always significant. Note also that 31 out of the 32 correlations for the summed similarity toward 10 exemplars were significant, while only 25 of the corresponding correlations for the prototype predictor reached significance. We will now focus on the four different dependent variables separately.

The typicality ratings, which could better be predicted than any of the other dependent variables, were significantly predicted by both predictors for all eight concepts. The ratings showed a considerably higher correlation with the exemplar predictor for fruits, vehicles, and fish, but the prototype predictor explained the typicalities of furniture better. For the remaining concepts, the difference between the two predictors was rather small. On the average, the difference between the two predictors in explaining typicality was only marginally significant. When predicting the typicalities of the whole item set, that is, including related nonmembers, the prediction based on both measures increased considerably, but no significant difference between the two predictors could be observed.

As to the response times in a speeded categorization task, the exemplar-based measure was also a better predictor for fruits and vehicles, whereas the prototype predictor better accounted for the response times for kitchen utensils. Averaged over the eight concepts, the difference between the two predictors in predicting the response times was again not significant.

Looking at the correlations for the categorynaming task, the exemplar-based predictor was better for fruits, fish, and vegetables, but the prototype measure was better for sports and furniture. Averaged over the eight concepts, the better predictions of the exemplar-based measure were not significantly different from the prototype predictions. The results of the reverse process though, where subjects were given the concept label and were asked to generate exemplars, were significantly better predicted by the 
exemplar-based measure. Only for furniture the prototype predictor did as good as the exemplarbased predictor. A possible explanation for the clearly better exemplar-based predictor for these data lies in the similarity of the method for gathering data: Both the exemplar-based predictor and the exemplar-generation frequencies start from production frequencies.

The analyses also showed that the results of the different concepts, regardless of the task, were predictable to a different extent. The data for birds and for vehicles could be predicted rather well, while the data for fruits, vegetables, and kitchen utensils seemed to resist prediction most. The differences, however, were not significant.

The reader may also recall that several prototype predictors were calculated and compared. The best prototype prediction was a simple unweighted sum of the applicability frequencies, summed over all features. This does not support the suggestion of Rosch and Mervis (1975) that the features possessed by the different items are weighted by their cue validity to determine degree of category membership. The results are in line, however, with findings of Hampton (1979), who also tried two different sorts of feature weighting, production frequency and rated feature importance, and found that none of these weightings improved the prediction of typicality ratings. Our findings that weighting did not improve the prototype prediction thus confirm Hampton's results.

The prototype measure in this first experiment was derived using the same procedure used by Hampton (1979). He used the prototype measure to predict responses in a speeded categorization task. In general, the corresponding correlations obtained in Experiment 1 were somewhat higher than the values reported by Hampton. Especially for kitchen utensils, the difference was considerable, since Hampton's correlation for this concept was rather low. The higher values obtained in our experiment reassure us of the soundness of the response times from our speeded categorization task as well as of the prototype predictor.

However, different procedures have been proposed in the literature to calculate prototype predictors. Essentially, the difference between a prototype measure and an exemplar-based measure is that the former is based on one representation for the whole concept, while the latter is based on many representations-one for every stored exemplar - which are summarized or averaged. Assuming that the representations consist of features or attributes, we can rephrase this by stating that the prototype predictors are based on one single vector of attribute values, while exemplar predictors are based on the average of many different vectors of attribute values. The different procedures to calculate prototype predictors differ in the way to obtain the attributes of which the concept vector of attribute values consists. Hampton's (1979) procedure, which was used in Experiment 1, gathered these attributes starting from the category label, by simply asking participants to generate features of the concept. ${ }^{2}$ Since the results of Experiment 1 showed that the exemplar-based measure predicted the dependent variables a little better than Hampton's prototype procedure, another experiment was conducted to evaluate how consistent this advantage of the exemplar predictor was when varying the procedure to derive the prototype. Experiment 2 was set up to test another procedure, based on Rosch \& Mervis (1975) classical family resemblance procedure.

\section{EXPERIMENT 2}

Rosch and Mervis (1975, p. 575) have described the prototype structure of semantic categories as follows: "the basic hypothesis was that members of a category come to be viewed as prototypical of the category as a whole in proportion to the extent to which they bear a

${ }^{2}$ According to Hampton (1979), when asked to give features of a concept, participants are assumed to be able to activate this information directly, without first instantiating exemplars of the concept. This procedure can therefore be criticized because exactly what goes on in a subject's mind when he or she is asked to give features of a concept is not directly observable. It is very well conceivable that subjects instantiate exemplars, activate features of these exemplars, and respond with those features that apply to enough of the instantiated exemplars. We thank Larry Barsalou for pointing this out. However, if the activation of these "concept features" really happens after exemplar instantiation, one should still assume that participants first apply a sort of filter, since no features idiosyncratic for just one (or a few) exemplars are given. 
family resemblance to (have attributes which overlap those of) other members of the category." While this description gives a generally accepted conception of a prototype of a semantic category, it is not clear at all how the relevant attributes on which the prototype is based can be obtained empirically. For an elaborate discussion of this difficulty, see Smith \& Medin (1981).

The solution used by Hampton (1979), which we adopted in Experiment 1, is to simply ask subjects to generate features that characterize the concepts under study. More in detail, Hampton asked participants to give descriptions of the eight categories. For each category, a set of seven different questions was used in order to encourage the participants to generate as many different properties as they could. These questions were, for example, why some items only "loosely speaking" belong to a particular category, or why a certain item might be considered a very typical example of the category. Importantly, in all questions involving the consideration of particular examples, no specific example was ever given by the experimenter to avoid biasing the participants.

In Experiment 2, a prototype measure was computed using another procedure, which is more in line with the prototype theory as formulated by Rosch (1975a, 1975b, 1977, 1978), 1983). The procedure of Rosch and Mervis (1975) was applied, where the attributes of the category were gathered starting from the exemplars of the category. Next, for each category, all attributes of the exemplars were listed and judged for their applicability for every exemplar. Each attribute then received a weight, based on the number of items that had been credited with that attribute. Finally, a family resemblance score could be calculated for every item by summing the weights of the attributes that were judged to apply to the item. (Another version of this new prototype measure was calculated, without weighting the different features. Averaged over the four dependent variables and the eight concepts, the unweighted version explained less variance than the weighted version, but like for the weighted and unweighted prototype versions in Experiment 1, the difference was not significant.)
Thus, the purpose of Experiment 2 is similar to the purpose of Experiment 1: Again the four dependent variables (typicality ratings, response times from the speeded categorization task, category-naming frequencies, and exemplar-generation frequencies) were correlated with the new family resemblance measure, which was then compared with the prototype measure based on Hampton's procedure and with the exemplarbased predictor of Experiment 1.

\section{Method}

Participants. There were six different participants in this experiment: Three of them participated in the attribute generation task and four judged the attribute applicabilities for every item. All participants were graduate students of the Psychology Department of the University of Leuven.

Material. The same eight concepts from Experiment 1 were used. Because of the very high correlations obtained for the typicality predictions including related nonmembers in Experiment 1 , and because of the time-consuming nature of the matrix filling task in the second experiment, data were gathered for the 24 concept members only (thus dropping the 12 related nonmembers of the item list).

Procedure. In the attribute generation task, three participants generated features for all 24 members of five or six concepts. For every concept, two different participants took part in the attribute generation task. They were given all 24 items of every concept together and they were instructed to write down as many attributes as possible for each of the items, but they did not have to repeat an attribute that was already written down for a previous item of the same concept.

In the attribute applicability judgment task, four different participants filled up all entries of the eight grids, each consisting of 24 rows for the items and 26 to 73 columns for the attributes. The participants were allowed to work on the task at different moments, but they were asked to complete a grid before pausing, once they started it. Completing a single grid took between 25 and 50 min. 


\section{Results}

Attribute generation task. As in the procedure described by Rosch and Mervis (1975), all attributes generated by any participant in the generation task for any of the 24 items were taken up in the attribute list for a concept. This resulted in 55, 55, 73, 53, 33, 37, 26, and 36 attributes for fruits, birds, vehicles, sports, furniture, fish, vegetables, and kitchen utensils, respectively.

Items by attributes matrix applicability task. Interscorer reliabilities were calculated by dividing the four participants at random in two groups of two. Next, for each of the eight matrices, the $0 / 1$ values in every cell were summed over the two raters of every group. Finally, the correlation between the two sum scores over all cells of the matrix of every concept was calculated and the Spearman-Brown formula for estimating the reliability was applied. This resulted in estimates of $.88, .93, .90, .91, .91, .92$, .92 , and .92 , respectively.

Calculating the family resemblance scores. Family resemblance scores were calculated following the procedure used by Rosch and Mervis (1975). The procedure described below is illustrated in Fig. 3. First, the corresponding cells in the item by feature applicability matrix were summed over the four judges. The resulting frequency matrix was dichotomized by changing all cell entries with a value of 3 or more into a 1 ; all other cells were given a 0 value. (In other words, if the majority of the judges indicated that a particular attribute applied to a certain item, than the cell value was 1 ; otherwise the cell value was 0 .) Next, each attribute received a weight, ranging from 1-24, representing the number of items in the category that had been credited with that attribute. Finally, the basic measure of degree of family resemblance for an item was the sum of the weighted scores of each of the attributes that had been credited to that item.

Table 4 shows the correlations of the family resemblance scores with the Hampton prototype measure and the exemplar-based measure (based on the 10 exemplars used in Experiment 1 ), and with each of the four dependent variables. The new prototype measure corresponds well to the prototype measure based on Hampton (1979) used in Experiment 1, but the two measures are not quite the same: All correlations are above .73 , but for only one of the eight concepts is the correlation as high as .90. The correlation of the new measure with the exemplar-based measure based on 10 instantiations is somewhat lower in general.

Concerning the prediction of the four dependent variables, the correlations of Table 4 are a little lower than the corresponding values of the Hampton prototype measure (see Table 1). As in Experiment 1, the new (family resemblance) prototype measure was compared with the exemplar-based measure of Experiment 1, but at the same time with Hampton's (1979) prototype measure, using an analysis of variance with a split-plot factorial design (Kirk, 1982), where the eight concepts function as blocks, where the predictor variables (Rosch's family resemblance, Hampton's prototype measures, and exemplar-based predictors) and the four dependent variables (typicality ratings, response times, category-naming, and exemplar-generation frequencies) are within-block factors, and where the sort of concepts (artifacts and natural kinds) is a between-block factor. The dependent variable in the ANOVA was the percentage of explained variance (the square of the correlations shown in the four last columns of Table 4 and in the second through the fifth column of Table 2).

The analysis yielded a significant effect of the predictor variable $(F(2,12)=5.19, p<.05)$. When comparing the exemplar-based measure with the two prototype measures in a single contrast, the exemplar-based measure yielded significantly better predictions $(t(12)=3.18$, $p<.01)$. The difference between the two prototype predictors was not significant. Furthermore, the dependent variable factor was also significant $(F(3,18)=15.19, p<.01)$. As in Experiment 1, the typicality ratings were predicted better than the results of the other three tasks $(t(18)=5.75, p<.01)$, the response times were better predicted than the exemplar-generation frequencies and the category-naming frequencies $(t(18)=3.02, p<.01)$, and the latter two did not differ significantly. Most importantly, the interaction effect of the predictor 
Step1: Filling up the Item by Feature Matrix

Features Generated for Individual Items

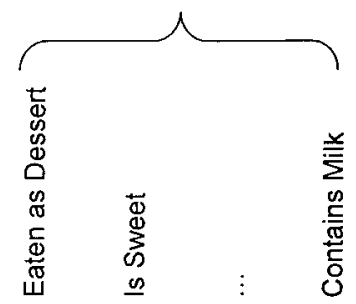

$\begin{array}{ccccc}\begin{array}{c}\text { Strawberry } \\ \text { Pineapple }\end{array} & 4 & 4 & \ldots & 0 \\ \vdots & & 4 & \ldots & 0\end{array} \quad \begin{aligned} & \text { Number of Subjects out of } 4 \text { that } \\ & \text { Credited "Coconut" with the Feature } \\ & \text { Coconut }\end{aligned}$

Step2: Dichotomizing the Frequency Matrix according to a Majority Rule and Calculating the Feature Weights Vector

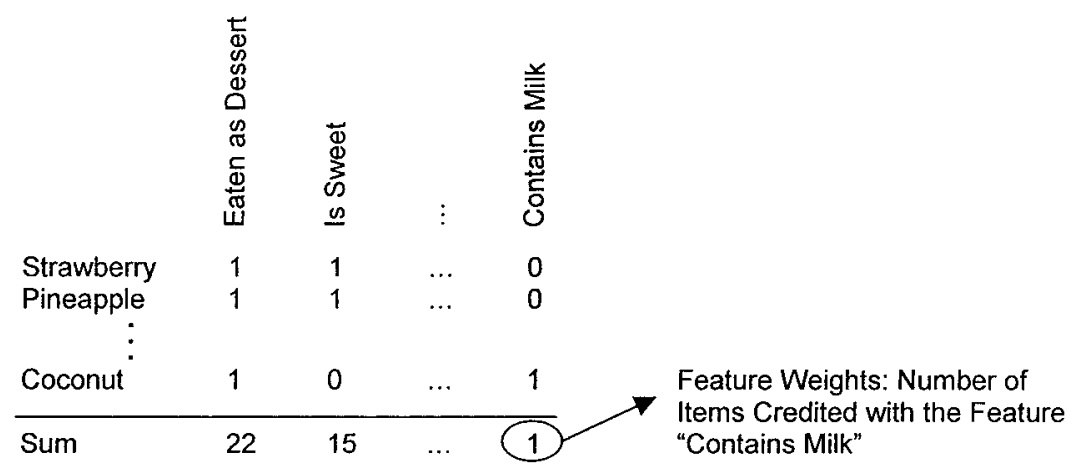

Step3: Post-Multiplying the Dichotomized Matrix with the Feature Weights Vector

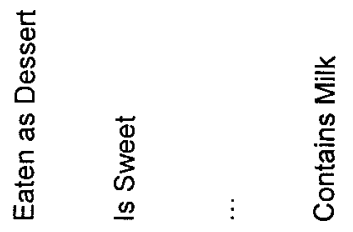

\begin{tabular}{lllll} 
Strawberry & 22 & 15 & $\ldots$ & 0 \\
Pineapple & 22 & 15 & $\cdots$ & 0 \\
\multicolumn{1}{c}{$\begin{array}{l}\text { Coconut } \\
\text { Sum }\end{array}$} & 22 & 0 & $\ldots$ & 1 \\
& 22 & 15 & $\ldots$ & 1
\end{tabular}

Step4: Calculating the Family Resemblance Predictor by Summing the Feature Weights of the Credited Features

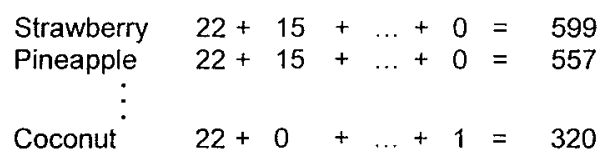

FIG. 3. Calculating the Rosch and Mervis (1975) family resemblance predictor. 
TABLE 4

Correlations of the Family Resemblance Scores for the Category Members with the Hampton Prototype Predictors, the Exemplar Predictors Based on Weighted Sum of Similarities Toward the Ten Most Frequently Generated Exemplars, and Each of the Four Dependent Variables

Correlations of the family resemblance scores versus

\begin{tabular}{|c|c|c|c|c|c|c|}
\hline Concept & $\begin{array}{l}\text { Hampton prototype } \\
\text { predictors }\end{array}$ & $\begin{array}{l}\text { Exemplar } \\
\text { predictors }\end{array}$ & Typicality & $\begin{array}{l}\text { Mean response } \\
\text { times }\end{array}$ & $\begin{array}{l}\text { Category-naming } \\
\text { frequencies }\end{array}$ & $\begin{array}{l}\text { Exemplar-generation } \\
\text { frequencies }\end{array}$ \\
\hline Fruits & $.77 * *$ & $.79 * *$ & $.76^{* *}$ & $-.47 *$ & $.52 * *$ & $.45^{*}$ \\
\hline Birds & $.82 * *$ & $.75 * *$ & $.80 * *$ & $-.68 * *$ & $.64 * *$ & $.38 *$ \\
\hline Vehicles & $.79 * *$ & $.86^{* *}$ & $.88 * *$ & $-.59 * *$ & $.60 * *$ & $.61 * *$ \\
\hline Sports & $.91 * *$ & $.71 * *$ & $.84 * *$ & $-.61 * *$ & $.66^{* *}$ & $.41 *$ \\
\hline Furniture & $.73 * *$ & $.76^{* *}$ & $.75 * *$ & $-.78 * *$ & $.51 * *$ & $.51 * *$ \\
\hline Fish & $.82 * *$ & $.50 * *$ & $.58 * *$ & $-.72 * *$ & $.38^{*}$ & .23 \\
\hline Vegetables & $.79 * *$ & $.52 * *$ & $.60 * *$ & $-.41 *$ & .21 & -.12 \\
\hline Kitchen utensils & $.80 * *$ & $.61 * *$ & $.56^{* *}$ & $-.51 * *$ & .18 & $.50 * *$ \\
\hline Mean & $.81 * *$ & $.71 * *$ & $.74 * *$ & $-.61 * *$ & $.48 * *$ & $.39^{*}$ \\
\hline
\end{tabular}

Note. ${ }^{*} p<.05 . * * p<.01$. All others not significant.

variable and the dependent variable factor was significant $(F(6,36)=4.21, p<.01)$. Contrasts were calculated to see whether there was a difference between the three predictor variables for each of the four dependent variables. For the typicality ratings, the exemplar-based predictor was significantly better than the average of the two prototype predictors $(t(36)=2.05, p<$ $.05)$. For the response times, the advantage of the exemplar-based predictor over the two prototype predictors was marginally significant $(t(36)=1.52, p<.10)$. For the exemplar generation frequencies, again the exemplar-based predictor was significantly better than the average of the two prototype predictors $(t(36)=$ $7.27, p<.01)$. Finally, for category naming, the advantage of the exemplar-based predictor was significant $(t(36)=1.91, p<.05)$. Furthermore, the difference between the two prototype predictors did not reach significance for any of the four dependent variables.

\section{Discussion}

The results obtained in Experiment 2 show that the classic family resemblance prototype measure suggested by Rosch and Mervis (1975) to account for typicality and response times does yield significant predictions of the dependent variables: Correlations with typicality rat- ings, response times, exemplar-generation frequencies, and category-naming frequencies were significant for $8,8,5$, and 6 concepts out of 8 , respectively. To our best knowledge, the latter two dependent variables have never been predicted using Rosch and Mervis' classical family resemblance measure. Besides, the correlation with response times given by Rosch and Mervis were obtained from artificial categories, not natural language concepts. The correlations with typicalities are somewhat lower than the values reported by Rosch and Mervis. It is not clear why this is the case. We used more items (24 instead of 20) and more judges (4 instead of 2) in calculating the family resemblance measure than Rosch and Mervis. The number of attributes used in calculating the family resemblance scores was not reported in their paper.

The most important finding of Experiment 2, however, is that our exemplar-based predictor turned out to yield significantly better predictions than Rosch and Mervis' (1975) classical prototype measure. Thus, the (weighted) summed similarity toward the 10 most frequently generated exemplars of a category does predict typicality, response times, exemplargeneration frequencies, and category-naming frequencies better than the family resemblance measure. Moreover, averaged over the two pro- 
totype predictors, the exemplar-based measure yields significantly better predictions for all dependent variables, except for response times, where the difference was marginally significant.

As in Experiment 1, the typicality ratings could be predicted better than any of the other dependent variables, and the response times were also better predicted than the remaining two dependent variables, for which predictions did not differ significantly.

The analyses again show that the results of the different concepts, averaged over the four dependent variables, were predictable to a different extent. The percentage of explained variance differs over the concepts from 54 to $22 \%$. The data for birds and for vehicles can be predicted rather well, while the data for fruits and vegetables are not so well predictable. However, in the ANOVA, these differences were not significant.

\section{GENERAL DISCUSSION}

In this article, the results of two experiments were reported in which typicality ratings, response times (gathered in a speeded categorization task), category-naming frequencies, and exemplar-generation frequencies of natural language concepts were related to two sorts of predictors: prototype-based measures and an exemplar-based measure. The prototype measures were based on the idea that natural language concepts are represented in semantic memory by a set of features that are not strictly necessary and sufficient conditions for concept membership, but that are characteristic for items belonging to the category. Importantly, it is assumed that the concept is represented with a single vector of attribute values that is assumed to be characteristic for the whole category. The two prototype measures differed in the procedure used to empirically obtain this concept attribute vector from which predictions for individual items were derived.

First, according to Hampton (1979), an essential assumption of the prototype view is that these characteristic features are stored at the level of the concept and that they are directly accessible. Consequently, the procedure used to derive predictions described in Hampton (1979) consists in eliciting these directly stored at- tributes and to base the prediction on the extent to which items can be credited with these concept attributes or not.

Second, Rosch and Mervis' (1975) prototype measure is directly related to Wittgenstein's (1953) ideas about concepts, where a family resemblance relationship exists among a set of items and where each item has at least one, and probably several, elements in common with one or more other items, but no, or few, elements are common to all items. Natural language concepts are viewed as networks of overlapping attributes and members of a category are viewed as prototypical to the extent that they have attributes in overlap with other members of the category. The procedure used to obtain the prototype vector of concept attributes (and the corresponding weights) therefore starts from attributes generated with concept exemplars as cues, not with the concept label as cue.

Note that for the Hampton prototype, weighting of the features based on production frequency (or importance ratings) yielded predictions that were somewhat worse than no weighting at all, while for the Rosch and Mervis prototype, weighting did yield better predictions. The difference between weighted and unweighted versions, however, was not significant for any of the two procedures.

Both prototype measures were compared with an exemplar-based prediction. The results of the reported experiments show that the exemplar-based measure predicted the four dependent variables significantly better than the prototype models. However, essentially, in comparing exemplar-based measures with prototype measures, one compares the similarity of a target item with a single vector that is assumed to be representative for the whole concept with the similarity of the target item with multiple vectors, one for every exemplar. Since the similarity of the target item to the attribute vectors of all activated exemplars are usually assumed to be combined (by, for instance, an averaging process, possibly after weighting) one can ask the question whether prototype and exemplarbased predictors can be differentiated at all (Barsalou, 1990). Theoretically, one might expect both measures to yield the same information if the prototype predictor is constructed in 
the first place from (1) exactly the same set of stored exemplars and (2) the same fixed set of attributes of exemplars on which the exemplar predictor is based. Some of our results, however, suggest that this is not the case.

First, the correlations between the prototype and the exemplar-based predictors that we empirically obtained indicate that both measures are not at all the same, even when taking into account the (un)reliability of the measures. Only for birds and vehicles high correlations were obtained. For some concepts, the two measures have only $25 \%$ of their variance in common. These results suggest that our empirically obtained exemplar measure contains information that is not contained in the prototype measures.

Second, the prototype view assumes that a concept's prototype is constructed using information on all exemplars of the concept. In Rosch and Mervis (1975), a sample of items which is assumed to be representative of the whole concept is used to derive the family resemblance measure. Hampton $(1979,1993)$ also makes similar assumptions about how prototypes are formed. Our data, however, clearly suggest that the exemplar prediction does not improve or even gets worse when taking into account more than the ten most frequently generated exemplars.

Third, in order to determine where the surplus value of the exemplar predictor is situated, another exemplar-based measure was calculated. The new measure was based on the summed similarity toward the same generated exemplars of the category, but the similarities were not rated similarities, but calculated similarities based on the characteristic features of the Hampton prototype. In analytic terms, the new predictor variable was constructed by calculating the distance function

$$
d_{i}=\sum_{n=1}^{N} \sqrt{\sum_{m=1}^{M}\left(x_{i m}-x_{n m}\right)^{2}},
$$

where $i$ indexes the test item, $x_{i m}$ refers to the applicability of the $m$ th characteristic feature of the concept to the test item $i$, and $x_{n m}$ refers to the applicability of the $m$ th feature to the $n$th most frequently generated exemplar of the category. To avoid a too lengthy Results section, we only report the correlations of the typicality ratings with the sum of the calculated similarities toward the 10 most frequently generated exemplars. These correlations were $-.66,-.74$, $-.54,-.74,-.82,-.79,-.69$, and -.62 , for the concepts fruits, birds, vehicles, sports, furniture, fish, vegetables, and kitchen utensils, respectively. (Note that the correlations with a distance measure correlate negative instead of positive.) The correlations follow the predictions of Hampton's prototype predictor rather well for fruits, furniture, fish, vegetables, and kitchen utensils. For sports, the results are closer to the prediction of the exemplar model. For birds and vehicles, the predictions of this alternative exemplar model are clearly inferior to both Hampton's prototype predictions, the predictions of the exemplar model based on the direct similarity ratings, and to the family resemblance predictions. Similar measures were calculated based on the complete feature set on which the family resemblance measure of Experiment 2 was based. The results were again less predictive than the exemplar measure based on directly rated similarities.

These results clearly show that the rated similarities used in the exemplar-based measure differ from the calculated similarities based on characteristic features of the concept, as gathered by Hampton (1979) or in the family resemblance measure (Rosch \& Mervis, 1975). Most likely, the rated similarities are richer and are based on more or other features. Possibly the similarities are based on different feature sets depending on the specific activated exemplar and target item considered. As Medin, Goldstone, and Gentner (1993) argued similarity is a useful concept if one can specify the ways or respects in which things are similar and the respects are determined by processes internal to comparisons. This implies that the respects are not necessarily the respects highlighted by Hampton's characteristic features. At any rate, the results clearly showed that the exemplar measure based on the rated similarities yielded better predictions, that is, rated similarities account better for the four dependent variables 
than the similarities based on the characteristic features of the concept.

It is important to note that the two prototype models used in our experiments are based on sums of feature applicabilities, possibly weighted for feature frequencies (or importance ratings). None of the prototype models assumes that correlations among features are stored and used in category-related decisions. As Barsalou (1990) argues, abstraction models that do take into account feature correlations are very well conceivable. As far as we know, such models have not yet been applied to natural language concepts.

We will conclude this article with some comments on category learning versus natural language concepts. To our best knowledge, this is the first study in which predictions of the prototype view and of the exemplar view are compared to predict category-based decisions for natural language concepts. Our results differ somewhat from the findings in the majority of papers that have investigated category learning, where it is assumed that all previously encountered exemplars of a category are stored and are activated whenever category-related decisions have to be made (Gluck \& Bower, 1988; Medin, Altom, \& Murphy, 1984; Medin, Dewey, \& Murphy, 1983; Medin \& Schaffer, 1978; Medin \& Smith, 1981; Nosofsky, 1987, 1988, 1991). The data presented here suggest that activation of a limited number of frequently generated exemplars of the studied concepts suffices to predict typicality ratings, response times, exemplar-generation frequencies, and category-naming frequencies significantly and that elaborating the exemplar set can even decreases the predictive power. This discrepancy between the results of the studies with natural language concepts and with artificial categories may be attributable at least partly to the differences in the learning situations. There are at least two major differences between the category learning situations studied by Gluck and Bower, Medin and his colleagues, and Nosofsky, on the one hand, and the way natural language concepts are learned and represented on the other hand. First, as Malt and Smith (1984) quite rightly pointed out, participants in category learning tasks are instructed to encode the exemplars in detail in an explicit learning phase, while natural language concepts are learned erratically and from many different sources. Second, all natural language concepts used in our study (fruit, birds, furniture, etc.) are common concepts that are probably learned in early childhood. The participants in our typicality-rating task and in the speeded categorization task were all adult language users who have been using these concepts for many years. It is possible that knowledge about these common and frequently used categories is reorganized into a different representation over time, where only the best exemplars are activated by the concept label. Keep in mind, however, that our results are not perfectly comparable with the results from most category learning experiments, since the dependent variable in the latter studies (classification decisions) differs from the dependent variables used in our study.

The findings for natural language concepts reported here are in line with the literature on category learning experiments in that the exemplar-based predictor accounts better for the four dependent variables than Hampton's and Rosch and Mervis' prototype measures. (For a detailed comparison between exemplar and prototype models in category learning, see Nosofsky, 1992).

Finally an important difference between the two experiments on natural language concepts reported here and most category learning experiments concerns the dependent variable studied. The dependent variable to be explained usually in the category learning experiments refers to classification decisions of new exemplars that have not been presented previously. In the experiments described in this paper, though, the criterion variables that are predicted are not classification decisions, but typicality ratings, response times in a speeded categorization task, category-naming frequencies, and exemplargeneration frequencies. Furthermore, the stimuli were well-known exemplars of familiar concepts. Relevant findings about classification decisions of unfamiliar stimuli in well-known natural language concepts can be found in Storms, De Boeck, and Ruts (1998). 


\section{APPENDIX}

Ten Most Frequently Generated Exemplars for Every Category Studied

\begin{tabular}{|c|c|c|c|c|c|c|c|c|}
\hline Rank & Fruits & Birds & Vehicles & Sports & Furniture & Fish & Vegetables & Kitchen utensils \\
\hline 1 & apple & sparrow & car & soccer & chair & goldfish & lettuce & knife \\
\hline 2 & banana & parrot & bicycle & volleyball & table & cod & carrots & pot \\
\hline 3 & pear & robin & airplane & tennis & bed & salmon & $\begin{array}{l}\text { tomatoes } \\
\text { red }\end{array}$ & pan \\
\hline 4 & orange & eagle & train & basketball & cupboard & plaice & cabbage & spoon \\
\hline 5 & kiwi & blackbird & streetcar & swimming & seat & eel & cauliflower & fork \\
\hline 6 & peach & canary & bus & ski & night table & trout & leek & blender \\
\hline 7 & grape & pigeon & boat & tabletennis & $\begin{array}{l}\text { bookcase } \\
\text { dumb }\end{array}$ & makrel & peas & ladel \\
\hline 8 & cherry & ostrich & moped & hockey & waiter & sole & chicory & chopping board \\
\hline 9 & strawberry & seagull & lorry & badminton & coffee table & pike & beans & plate \\
\hline 10 & pineapple & woodpecker & motorcycle & athletics & desk & herring & cucumber & stove \\
\hline
\end{tabular}

\section{REFERENCES}

Barsalou, L. W. (1990). On the indistinguishability of exemplar memory and abstraction in category representation. In T. K. Srull \& R. S. Wyer (Eds.), Advances in social cognition: Content and process specificity in the effects of prior experiences (Vol. 3, pp. 61-88). Hillsdale, NJ: Erlbaum.

Barsalou, L. W., Huttenlocher, J., \& Lamberts, K. (1998). Basing categorization on individuals and events. Cognitive Psychology, 36, 203-272.

Gluck, M. A., \& Bower, G. H. (1988). From conditioning to category learning: An adaptive network model. Journal of Experimental Psychology: General, 117, 227247.

Hampton, J. A. (1979). Polymorphous concepts in semantic memory. Journal of Verbal Learning and Verbal Behavior, 18, 441-461.

Hampton, J. A. (1993). Prototype models of concept representations. In I. Van Mechelen, J. A. Hampton, R. S. Michalski, \& P. Theuns (Eds.), Categories and concepts: Theoretical views and inductive data analysis (pp. 67-95). London: Academic Press.

Hampton, J. A., \& Gardiner, M. M. (1983). Measures of internal category structure: A correlational analysis of normative data. British Journal of Psychology, 74, $498-516$.

Hayes-Roth, B., \& Hayes-Roth, F. (1977). Concept learning and the recognition and classification of exemplars. Journal of Verbal Learning and Verbal Behavior, 16, 321-338.

Heit, E., \& Barsalou, L. W. (1996). The instantiation principle in natural language categories. Memory, 4, 413451.

Hines, D., Czerwinski, M., Sawyer, P. K., \& Dwyer, M. (1986). Automatic semantic priming: Effect of category exemplar level and word association level. Journal of Experimental Psychology: Human Perception and Performance, 12, 370-379.

Keller, D., \& Kellas, G. (1978). Typicality as a dimension of encoding. Journal of Experimental Psychology: Human Learning and Memory, 4, 78-85.

Kirk, R. E. (1982). Experimental design: Procedures for the behavioral sciences. Belfort, CA: Brooks/Cole.

Komatsu, L. K. (1992). Recent views of conceptual structure. Psychological Bulletin, 112, 500-526.

Larochelle, S., \& Pineau, H. (1994). Determinants of response times in the semantic verification task. Journal of Memory and Language, 33, 796-823.

Malt, B. C., \& Johnson, E. C. (1992). Do artifacts have cores? Journal of Memory and Language, 31, 195217.

Malt, B. C., \& Smith, E. E. (1984). Correlated properties in natural categories. Journal of Verbal Learning and Verbal Behavior, 23, 250-269.

McCloskey, M., \& Glucksberg, S. (1979). Decision processes in verifying category membership statements: Implications for models of semantic memory. Cognitive Psychology, 11, 1-37.

Medin, D. L. (1986). Comments on "Memory storage and retrieval processes in category learning." Journal of Experimental Psychology: General, 115, 373-381.

Medin, D. L., Altom, M. W., Edelson, S. M., \& Freko, D. (1982). Correlated symptoms and simulated medical classification. Journal of Experimental Psychology: Learning, Memory, and Cognition, 8, 37-50.

Medin, D. L., Altom, M. W., \& Murphy, T. D. (1984). Given versus induced category representations: Use of prototype and exemplar information in classification. Journal of Experimental Psychology: Learning, Memory, and Cognition, 10, 333-352.

Medin, D. L., Dewey, G. I., \& Murphy, T. D. (1983). Relationships between item and category learning: Evidence that abstraction is not automatic. Journal of Experimental Psychology: Learning, Memory, and Cognition, 9, 607-625.

Medin, D. M., Goldstone, R. L., \& Gentner, D. (1993). Respects for similarity. Psychological Review, 100, 254-278. 
Medin, D. M., \& Schaffer, M. M. (1978). Context theory of classification learning. Psychological Review, 85, 207238.

Medin, D. L., \& Smith, E. E. (1981). Strategies and classification learning. Journal of Experimental Psychology: Learning, Memory, and Cognition, 7, 241-253.

Nosofsky, R. M. (1987). Attention and learning processes in the identification and categorization of integral stimuli. Journal of Experimental Psychology: Learning, Memory, and Cognition, 13, 87-109.

Nosofsky, R. M. (1988). Exemplar-based accounts of relations between classification, recognition, and typicality. Journal of Experimental Psychology: Learning, Memory, and Cognition, 14, 700-708.

Nosofsky, R. M. (1991). Tests of an exemplar model for relating perceptual classification and recognition memory. Journal of Experimental Psychology: Human Perception and Performance, 17, 3-27.

Nosofsky, R. M. (1992). Exemplars, prototypes, and similarity rules. In A. F. Healy, S. M., Kosslyn, \& R. M. Shiffrin (Eds.), From learning theory to connectionist theory: Essays in honor of William K. Estes (Vol. 1, pp. 149-167). Hillsdale, NJ: Erlbaum.

Nosofsky, R. M., \& Palmeri, T. J. (1997). An exemplarbased random walk model of speeded classification. Psychological Review, 104, 266-300.

Reed, S. K. (1972). Pattern recognition and categorization. Cognitive Psychology, 3, 382-407.

Rips, L. J. (1975). Inductive judgments about natural categories. Journal of Verbal Learning and Verbal Behavior, 14, 665-681.

Rosch, E. (1975a). Cognitive reference points. Cognitive Psychology, 7, 532-547.

Rosch, E. (1975b). Cognitive representations of semantic categories. Journal of Experimental Psychology, 104, 192-233.

Rosch, E. (1977). Human categorization. In N. Warren
(Ed.), Studies in cross-cultural psychology (Vol. 1, pp. 1-49), London: Academic Press.

Rosch, E. (1978). Principles of categorization. In E. Rosch \& B. B. Lloyd (Eds.), Cognition and categorization (pp. 27-48). Hillsdale, NJ: Erlbaum.

Rosch, E. (1983). Prototype classification and logical classification: The two systems. In E. K. Scholnick (Ed.), New trends in conceptual representation: Challenges to Piaget's theory? (pp. 73-86). Hillsdale, NJ: Erlbaum.

Rosch, E., \& Mervis, C. B. (1975). Family resemblances: Studies in the internal structure of categories. Cognitive Psychology, 7, 573-605.

Smith, E. E., \& Medin, D. M. (1981). Categories and concepts. Cambridge, MA: Harvard University Press.

Storms, G., De Boeck, P., \& Ruts, W. (1998). Exemplar based predictions for categorization of unknown stimuli in well-known natural concepts: A case study. Manuscript submitted for publication.

Storms, G., De Boeck, P., Van Mechelen, I., \& Ruts, W. (1996). The dominance effect in concept conjunctions: Generality and interaction aspects. Journal of Experimental Psychology: Learning, Memory, and Cognition, 22, 1-15.

Storms, G., De Wilde, E., De Boeck, P., \& Ruts, W. (1999). The instantiation principle revised. Manuscript in preparation.

Sutcliffe, J. P. (1993). Concepts, class, and category in the tradition of Aristotle. In I. Van Mechelen, J. A. Hampton, R. S. Michalski, \& P. Theuns (Eds.), Categories and concepts: Theoretical views and inductive data analysis (pp. 35-65). London: Academic Press.

Wittgenstein, L. (1953). Philosophical investigations. New York: Macmillan.

(Received August 5, 1998)

(Revision received May 21, 1999) 